\title{
Solvability of Boundary Value Problems for Second Order Impulsive Differential Equations on Whole Line with a Non-Carathédory Nonlinearity
}

\author{
XiaOHu Yang AND Yuji LiU*
}

\begin{abstract}
We study a class of boundary value problems of the impulsive differential equations on whole lines with a non-Carathéodory nonlinearity. Sufficient conditions to guarantee the existence of solutions are established. A new Banach function space $X$ and its relatively compact property of subset of $X$ is proved. An example is given to illustrate the main results.
\end{abstract}

\section{INTRODUCTION}

The asymptotic theory of ordinary differential equations is an area in which there is great activity among a large number of investigators. In this theory, it is of great interest to investigate, in particular, the existence of solutions with prescribed asymptotic behavior, which are global in the sense that they are solutions on the whole line (or half line). The existence of global solutions with prescribed asymptotic behavior is usually formulated as the existence of solutions of boundary value problems on the whole line (or half line).

These problems arise from real world applications. For example, some chemical and biological systems can be modelled by an autocatalytic process (see, e.g. [23, 21]. In many of these process the system can support propagating wavefronts due to a combination of a reaction effect and a molecular diffusion. The pioneering model in this framework is due to Fisher, [20], who suggested the equation $u_{t}=u_{x x}+u(1-u)$ for studying the spatial spread of a favored gene in a population. The simplest generalization of that equation is the so called Fisher-Kolmogorov's equation $u_{t}=u_{x x}+f(u)$, where $f$ is a given function with two zeroes, say $u=0$ and $u=1$, and positive on $(0,1)$

2010 Mathematics Subject Classification. Primary: 34B37, 34B40, 34B16.

Key words and phrases. Second order impulsive differential equation, boundary value problem, non-Carathéodory nonlinearity, fixed point theorem.

${ }^{*}$ Supported by the National Natural Science Foundation of China (No: 11401111), the Natural Science Foundation of Guangdong province (No:S2011010001900) and the Natural science research project for colleges and universities of Guangdong Province (No: 2014KTSCX126). 
so that $u=0$ and $u=1$ are the only two stationary states of this equation. Equations like this one arises in many problems suggested, for instance, by the classical theory of population genetics or by certain flame propagation problems in chemical reactor theory (see, e.g. [6]). A travelling wavefront or travelling wave solution (t.w.s., in short) of this equation is a solution $u(t, x)$ having a constant profile, that is, such that $u(t, x)=\phi(x-c t)$, for some fixed $\phi(\xi)$ (called the wave shape) and a constant $c$ (called the wave speed). Specially important for the applications are t.w.s. connecting the two stationary states, $u=0$ and $u=1$.

A simple calculation shows that if $u(t, x)=\phi(x-c t)$ is a t.w.s. of $u_{t}=$ $u_{x x}+f(u)$, then the wave shape $\phi$ is a solution of the ODE $u^{\prime \prime}+c u^{\prime}+f(u)=0$. When a t.w.s. connects the stationary states, its corresponding wave shape is a positive heteroclinic solution of $u^{\prime \prime}+c u^{\prime}+f(u)=0$ that connects the equilibria 1 and 0 , that is, a solution of $u^{\prime \prime}+c u^{\prime}+f(u)=0$ defined on $R$ and satisfying $u(t) \in(0,1)$ for all $t \in \mathbb{R}$ and $\lim _{t \rightarrow-\infty} u(t)=1$ and $\lim _{t \rightarrow+\infty} u(t)=0$, see [1]. Thus it comes the following boundary value problem:

$$
\left(e^{c t} u^{\prime}\right)^{\prime}+e^{c t} f(u)=0, t \in \mathbb{R}, \lim _{t \rightarrow-\infty} u(t)=1, \lim _{t \rightarrow+\infty} u(t)=0 .
$$

It is well known that the homogeneous and non-homogeneous Robin boundary value problems of the Lane-Emden equations are as follows, respectively,

$$
\begin{aligned}
& -\Delta u(x)=u^{p}(x), x \in \Omega, \frac{\partial u}{\partial \vec{n}}+b(x) u=0 \text { on } \partial \Omega, \\
& -\Delta u(x)=u^{p}(x)+\lambda f(x), x \in \Omega, \frac{\partial u}{\partial \vec{n}}+b(x) u=0 \text { on } \partial \Omega,
\end{aligned}
$$

where $\Omega$ is a domain in the n-dimensional Euclidean space $R^{n}, \Delta=\sum_{i=1}^{n} \frac{\partial^{2}}{\partial x_{i}^{2}}$. Lane-Emden equations arise naturally from the study of various nonlinear phenomena, such as pattern formation, population evolution, chemical reaction and has attracted considerable attention in recent years [27, 29, 34].

One can see that the one-dimensional Robin boundary value problems of the Lane-Emden equations on whole line are as follows:

$$
\begin{aligned}
x^{\prime \prime}(t)+[x(t)]^{p} & =0, \quad t \in \mathbb{R}, \\
\lim _{t \rightarrow-\infty} x^{\prime}(t)-\alpha \lim _{t \rightarrow-\infty} x(t) & =0, \\
\lim _{t \rightarrow+\infty} x^{\prime}(t)+\beta \lim _{t \rightarrow+\infty} x(t) & =0, \\
x^{\prime \prime}(t)+[x(t)]^{p}+\lambda f(t) & =0, \quad t \in \mathbb{R}, \\
\lim _{t \rightarrow-\infty} x^{\prime}(t)-\alpha \lim _{t \rightarrow-\infty} x(t) & =0, \\
\lim _{t \rightarrow+\infty} x^{\prime}(t)+\beta \lim _{t \rightarrow+\infty} x(t) & =0 .
\end{aligned}
$$

In recent years, the existence of solutions of boundary value problems of the differential equations governed by nonlinear differential operator $\left[\Phi\left(u^{\prime}\right)\right]^{\prime}$ 
$=\left[\left|u^{\prime}\right|^{p-2} u^{\prime}\right]^{\prime}$ has been studied by many authors, see $[7,12,13,21,22,9,11$, $10,14,16,17]$.

Impulsive differential equation is one of the main tools to study the dynamics of processes in which sudden changes occur. The theory of impulsive differential equation has recently received considerable attention, see $[30,31,32]$. However, the study on existence of positive solutions of nonlocal boundary value problems for impulsive differential equations on whole real line has not been sufficiently developed $[2,3,5,4,8,9,26]$.

In all above mentioned papers, the boundary conditions are subjected to the two end points 0 and $+\infty$ (or $-\infty$ and $+\infty$ ) and the solutions obtained are defined on $[0,+\infty)$ (or $R$ ). An interesting question occurs: when one subjects the boundary conditions on two intermediate points $\xi, \eta$, how can we get solutions defined on $R$ of a boundary value problem of differential equations on whole line? This is the first motivation of the present paper.

On the other hand, in known papers [10,11, 12, 13, 14, 16, 17, 18, 33], concerning the differential equations $\left[\Phi\left(\rho(t) x^{\prime}(t)\right)\right]^{\prime}+f\left(t, x(t), \rho(t) x^{\prime}(t)\right)=0$, it is supposed that $(t, u, v) \rightarrow f(t, u, \rho(t) v)$ is a Carathéodory function, i.e.,

(i) $t \rightarrow f(t, u, \rho(t) v)$ is integral on $R$ for each $(u, v) \in \mathbb{R}^{2}$;

(ii) $(u, v) \rightarrow f(t, u, \rho(t) v)$ is continuous for almost all $t \in \mathbb{R}$;

(iii) for each $r>0$ there exists a integral function $\phi_{r}: R \rightarrow \mathbb{R}$ such that $|f(t, u, \rho(t) v)| \leq \phi_{r}(t)$ for almost all $t \in \mathbb{R}$ and $|u|,|v| \leq r$.

To the best of our knowledge, there has been no paper concerning the solvability of boundary value problem of $\left[\Phi\left(\rho(t) x^{\prime}(t)\right)\right]^{\prime}+f\left(t, x(t), \rho(t) x^{\prime}(t)\right)=$ $0, t \in=R$ with $(t, u, v) \rightarrow p(t) f(t, u, v)$ being a non-Carathéodory function: $t \rightarrow f(t, u, \rho(t) v)$ is not integral on $=R$.

Motivated by mentioned papers, to fill this gap, we consider the following boundary value problem for the impulsive singular differential equation on the whole line

$$
\left\{\begin{array}{l}
{\left[\Phi\left(\rho(t) x^{\prime}(t)\right)\right]^{\prime}+p(t) f\left(t, x(t), \rho(t) x^{\prime}(t)\right)=0, \text { a.e. } t \in \mathbb{R},} \\
x(\xi)=\int_{-\infty}^{+\infty} m(s) \phi\left(s, x(s), \rho(s) x^{\prime}(s)\right) d s, \\
\rho(\eta) x^{\prime}(\eta)=\int_{-\infty}^{+\infty} n(s) \psi\left(s, x(s), \rho(s) x^{\prime}(s)\right) d s, \\
\Delta x\left(t_{i}\right)=\lim _{t \rightarrow t_{i}^{+}} x(t)-x\left(t_{i}\right)=I\left(t_{i}, x\left(t_{i}\right), \rho\left(t_{i}\right) x^{\prime}\left(t_{i}\right)\right), i \in \mathbb{Z}, \\
\Delta \Phi\left(\rho\left(t_{i}\right) x^{\prime}\left(t_{i}\right)\right)=\lim _{t \rightarrow t_{i}^{+}} \Phi\left(\rho(t) x^{\prime}(t)\right)-\Phi\left(\rho\left(t_{i}\right) x^{\prime}\left(t_{i}\right)\right) \\
=J\left(t_{i}, x\left(t_{i}\right), \rho\left(t_{i}\right) x^{\prime}\left(t_{i}\right)\right), i \in \mathbb{Z},
\end{array}\right.
$$

where

(i) $\Phi(x)=|x|^{k-2} x$ with $k>1$, the inverse of $\Phi$ is denoted by $\Phi^{-1}$ and $\Phi^{-1}(x)=|x|^{l-2} x$ with $\frac{1}{k}+\frac{1}{l}=1$, 
(ii) $p: \mathbb{R} \rightarrow[0, \infty)$ with $p \in L_{l o c}^{1}(\mathbb{R})$ and $\int_{-\infty}^{0} p(t) d t=\int_{0}^{+\infty} p(s) d s=$ $+\infty$

(iii) $\rho: \mathbb{R} \rightarrow[0, \infty)$ with $\frac{\Phi^{-1}(\tau(\cdot))}{\rho(\cdot)} \in L_{l o c}^{1}(\mathbb{R})$ and $\int_{-\infty}^{0} \frac{\Phi^{-1}(\tau(u))}{\rho(u)} d u=$ $\int_{0}^{+\infty} \frac{\Phi^{-1}(\tau(u))}{\rho(u)} d u=+\infty$ with $\tau(t)=1+\left|\int_{\eta}^{t} p(s) d s\right|$,

(iv) $f, \phi, \psi: \mathbb{R}^{3} \rightarrow \mathbb{R}$ are weak Carathéodory functions (see Definition 2.1 in Section 2), $m, n \in L^{1}(\mathbb{R})$,

(v) $\mathbb{Z}=\{0, \pm 1, \pm 2, \cdots\},\left\{t_{i}: i \in \mathbb{Z}\right\}$ is a increasing sequence with $\lim _{i \rightarrow-\infty} t_{i}=-\infty$ and $\lim _{i \rightarrow+\infty} t_{i}=+\infty$,

(vi) $I, J:\left\{t_{i}: i \in \mathbb{Z}\right\} \times \mathbb{R}^{2} \rightarrow \mathbb{R}$ are discrete Carathéodory functions (see Definition 2.2 in Section 2).

The homogeneous form of boundary conditions in (1) is as follows: $x(\xi)=$ $0, \rho(\eta) x^{\prime}(\eta)=0$, which comes from a generalization of the boundary conditions $a \lim _{t \rightarrow-\infty} x(t)-b x(\xi)=c \lim _{t \rightarrow+\infty}(t)-d \rho(\eta) x^{\prime}(\eta)=0$. This kind of boundary conditions arise in the study of heat flow problems involving a bar of infinite length with two controllers at $t=-\infty$ and $t=+\infty$ adding or removing heat according to the temperatures and the change of temperature detected by two sensors at $t=\xi$ and $t=\eta$ respectively. But these two controllers do not work well. Problem (1) is also a generalization of the initial value problem, but the solutions gotten are defined on $\mathbb{R}$. One knows that $x^{\prime \prime}(t)=\left[1+x^{\prime}(t)\right]^{2}, t \in \mathbb{R}, x^{\prime}(0)=0, x(0)=1$ (corresponding to (1), $\Phi(x)=x, \rho(t)=1, p(t)=1$ and $f\left(t, x, x^{\prime}\right)=1+x^{\prime 2}, I=J=0$ and (i)-(vi) are satisfied) has solution $x(t)=-\ln |\cos t|$, it blowup at time $t= \pm \frac{\pi}{2}$. From this point, it is of interest and meaningful to study the existence of solutions of (1).

The purpose is to establish sufficient conditions for the existence of solutions of $\operatorname{BVP}(1)$. Since (ii) and $f$ is a weak Carathédory function, then $(t, u, v) \rightarrow p(t) f(t, u, \rho(t) v)$ is a non-Carathéodory function on $\mathbb{R}^{3}$. The remainder of this paper is organized as follows: the preliminary results are given in Section 2, the existence result of solutions of $\mathrm{BVP}(1)$ is proved in Section 3. Finally in Section 4, an example is given to illustrate the main results.

\section{Preliminary Results}

In this section, we present some background definitions. The preliminary results are given too. Denote

$$
\tau(t)=1+\left|\int_{\eta}^{t} p(s) d s\right|, \quad \sigma(t)=1+\left|\int_{\xi}^{t} \frac{\Phi^{-1}(\tau(u))}{\rho(u)} d u\right| .
$$

It is easy to show that $\sigma, \tau$ are continuous on $R$ and $\lim _{t \rightarrow \pm \infty} \sigma(t)=\lim _{t \rightarrow \pm \infty} \tau(t)=$ $+\infty$. 
Definition 2.1. $F: \mathbb{R} \times \mathbb{R} \times \mathbb{R} \rightarrow \mathbb{R}$ is called a weak Carathédory function, that is

(i) $(u, v) \rightarrow F\left(t, \sigma(t) u, \Phi^{-1}(\tau(t)) v\right)$ is continuous on $\mathbb{R}^{2}$ for a.e. $t \in \mathbb{R}$,

(ii) for each $r>0$, there exists nonnegative function $M_{r} \geq 0$ such that $|u|,|v| \leq r$ implies

$$
\left|F\left(t, \sigma(t) u, \Phi^{-1}(\tau(t)) v\right)\right| \leq M_{r} \text {, a.e.t } \in \mathbb{R} .
$$

Definition 2.2. $H:\left\{t_{i}: i \in Z\right\} \times \mathbb{R} \times \mathbb{R} \rightarrow \mathbb{R}$ is called a discrete Carathédory function, that is

(i) $(x, y) \rightarrow H\left(t_{s}, \sigma\left(t_{s}\right) x, \Phi^{-1}\left(\tau\left(t_{s}\right)\right) y\right)$ is continuous on $\mathbb{R}^{2}$ for all $s \in \mathbb{Z}$,

(ii) for each $r>0$, there exists nonnegative constants $M_{i r} \geq 0(i \in Z)$ such that $|x|,|y| \leq r$ implies

$$
\left|H\left(t_{s}, \sigma\left(t_{s}\right) x, \Phi^{-1}\left(\tau\left(t_{s}\right)\right) y\right)\right| \leq M_{s r}, s \in \mathbb{Z}, \sum_{s=-\infty}^{+\infty} M_{s r}<+\infty .
$$

Definition 2.3. Let $X$ be a real Banach space. An operator $T: X \rightarrow X$ is completely continuous if it is continuous and maps bounded sets into relatively compact sets.

Choose

$$
X=\left\{\begin{array}{c}
\left.x\right|_{\left(t_{s}, t_{s+1}\right]},\left.\rho x^{\prime}\right|_{\left(t_{s}, t_{s+1}\right]} \text { is continuous, } s \in \mathbb{Z}, \\
\text { and there exist the limits } \\
\lim _{t \rightarrow t_{s}^{+}} x(t), \lim _{t \rightarrow t_{s}^{+}} \rho(t) x^{\prime}(t), s \in \mathbb{Z}, \\
\lim _{t \rightarrow-\infty} \frac{x(t)}{\sigma(t)}, \lim _{t \rightarrow+\infty} \frac{x(t)}{\sigma(t)} \\
\lim _{t \rightarrow-\infty} \frac{\rho(t) x^{\prime}(t)}{\Phi^{-1}(\tau(t))}, \lim _{t \rightarrow+\infty} \frac{\rho(t) x^{\prime}(t)}{\Phi^{1}(\tau(t))}
\end{array}\right\} .
$$

For $x \in X$, define

$$
\|x\|=\max \left\{\sup _{t \in \mathbb{R}} \frac{|x(t)|}{\sigma(t)}, \sup _{t \in \mathbb{R}} \frac{\rho(t)\left|x^{\prime}(t)\right|}{\Phi^{-1}(\tau(t))}\right\} .
$$

Lemma 2.1. $X$ is a Banach space with $\|\cdot\|$ defined.

Proof. It is easy to see that $X$ is a normed linear space. Let $\left\{x_{u}\right\}$ be a Cauchy sequence in $X$. Then $\left\|x_{u}-x_{v}\right\| \rightarrow 0, u, v \rightarrow+\infty$. It follows that $x_{u} \in X$ and

$$
\begin{aligned}
& \sup _{t \in \mathbb{R}} \frac{\left|x_{u}(t)-x_{v}(t)\right|}{\sigma(t)} \rightarrow 0, u, v \rightarrow+\infty, \\
& \sup _{t \in \mathbb{R}} \frac{\rho(t)\left|x_{u}^{\prime}(t)-x_{v}^{\prime}(t)\right|}{\Phi^{-1}(\tau(t))} \rightarrow 0, u, v \rightarrow+\infty .
\end{aligned}
$$


So

$$
\begin{aligned}
& \sup _{t \in\left(t_{s}, t_{s+1}\right]} \frac{\left|x_{u}(t)-x_{v}(t)\right|}{\sigma(t)} \rightarrow 0, u, v \rightarrow+\infty, \lim _{t \rightarrow t_{s}^{+}} \frac{x_{u}(t)}{\sigma(t)} \text { exists, } s \in N, \\
& \sup _{t \in\left(t_{s}, t_{s+1}\right]} \frac{\rho(t)\left|x_{u}^{\prime}(t)-x_{v}^{\prime}(t)\right|}{\Phi^{-1}(\tau(t))} \rightarrow 0, u, v \rightarrow+\infty, \lim _{t \rightarrow t_{s}^{+}} \frac{\rho(t) x_{u}^{\prime}(t)}{\Phi^{-1}(\tau(t))} \text { exists, } s \in \mathbb{Z} .
\end{aligned}
$$

Then there exists functions $x_{s, 0}, y_{s, 0} \in C^{0}\left[t_{s}, t_{s+1}\right]$ such that $\lim _{u \rightarrow+\infty} \frac{x_{u}(t)}{\sigma(t)}=$ $x_{s, 0}(t)$ and $\lim _{u \rightarrow+\infty} \frac{\rho(t) x_{u}^{\prime}(t)}{\Phi^{-1}(\tau(t))}=y_{s, 0}(t)$ uniformly on $\left[t_{s}, t_{s+1}\right]$.

Define $x_{0}(t)=x_{s, 0}(t), y_{0}(t)=y_{s, 0}(t)$ for all $t \in\left(t_{s}, t_{s+1}\right](s \in N)$. Then $x_{0}, y_{0}: \mathbb{R} \rightarrow \mathbb{R}$ is well defined on $\mathbb{R}$ and

$$
\lim _{u \rightarrow+\infty} \frac{x_{u}(t)}{\sigma(t)}=x_{0}(t), \lim _{u \rightarrow+\infty} \frac{\rho(t) x_{u}^{\prime}(t)}{\Phi^{-1}(\tau(t))}=y_{0}(t), t \in \mathbb{R} .
$$

It follows that

$$
\begin{gathered}
\sup _{t \in\left(t_{s}, t_{s+1}\right]}\left|\frac{x_{u}(t)}{\sigma(t)}-x_{0}(t)\right| \rightarrow 0, u \rightarrow+\infty \\
\sup _{t \in\left(t_{s}, t_{s+1}\right]}\left|\frac{\rho(t) x_{u}(t)}{\Phi^{-1}(\tau(t))}-y_{0}(t)\right|, u \rightarrow+\infty .
\end{gathered}
$$

Step 1. Prove that $\sigma(\cdot) x_{0}(\cdot), \Phi^{-1}(\tau(\cdot)) y_{0}(\cdot) \in C^{0}\left(t_{s}, t_{s+1}\right]$ and the limits $\lim _{t \rightarrow t_{s}^{+}} \sigma(t) x_{0}(t)$ and $\lim _{t \rightarrow t_{s}^{+}} \Phi^{-1}(\tau(t)) y_{0}(t)$ exist.

We have for $\bar{t}_{0} \in\left(t_{s}, t_{s+1}\right]$ that

$$
\begin{aligned}
& \begin{aligned}
\left|\sigma(t) x_{0}(t)-\sigma\left(\bar{t}_{0}\right) x_{0}\left(\bar{t}_{0}\right)\right| & \leq\left|\sigma(t) x_{0}(t)-x_{N}(t)\right|+\left|x_{N}(t)-x_{N}\left(\bar{t}_{0}\right)\right| \\
& +\left|x_{N}\left(\bar{t}_{0}\right)-\sigma\left(\bar{t}_{0}\right) x_{0}\left(\bar{t}_{0}\right)\right|
\end{aligned} \\
& \begin{array}{r}
\leq 2 \sup _{t \in\left(t_{s}, t_{s+1}\right]} \sigma(t)\left|\frac{x_{N}(t)}{\sigma(t)}-x_{0}(t)\right|+\left|x_{N}(t)-x_{N}\left(t_{0}\right)\right| \\
\leq 2 \max _{t \in\left[t_{s}, t_{s+1}\right]} \sigma(t) \sup _{t \in\left(t_{s}, t_{s+1}\right]}\left|\frac{x_{N}(t)}{\sigma(t)}-x_{0}(t)\right|+\left|x_{N}(t)-x_{N}\left(t_{0}\right)\right| .
\end{array}
\end{aligned}
$$

Since $\sup _{t \in\left(t_{s}, t_{s+1}\right]}\left|\frac{x_{u}(t)}{\sigma(t)}-x_{0}(t)\right| \rightarrow 0, u \rightarrow+\infty$ and $x_{u}(t)$ is continuous on $\left(t_{s}, t_{s+1}\right]$, then for any $\epsilon>0$ we can choose $N$ and $\delta>0$ such that $\sup _{t \in\left(t_{s}, t_{s+1}\right]}\left|\frac{x_{N}(t)}{\sigma(t)}-x_{0}(t)\right|<\epsilon$ and $\left|x_{N}(t)-x_{N}\left(\bar{t}_{0}\right)\right|<\epsilon$ for all $\left|t-\bar{t}_{0}\right|<\delta$. Thus $\left|\sigma(t) x_{0}(t)-\sigma\left(\bar{t}_{0}\right) x_{0}\left(\bar{t}_{0}\right)\right|<3 \epsilon$ foe all $\left|t-\bar{t}_{0}\right|<\delta$. So $\sigma(\cdot) x_{0}(\cdot) \in$ $C^{0}\left(t_{s}, t_{s+1}\right]$. Similarly we can prove that $\Phi^{-1}(\tau(\cdot)) y_{0}(\cdot) \in C^{0}\left(t_{s}, t_{s+1}\right]$. We see easily that the limits $\lim _{t \rightarrow t_{s}^{+}} \sigma(t) x_{0}(t)$ and $\lim _{t \rightarrow t_{s}^{+}} \Phi^{-1}(\tau(t)) y_{0}(t)$ exist.

Step 2. Prove that the limits $\lim _{t \rightarrow \pm \infty} x_{0}(t), \lim _{t \rightarrow \pm \infty} y_{0}(t)$ exist. 
Suppose that $\lim _{t \rightarrow-\infty} x_{u}(t)=A_{u}^{-}$. By $\sup _{t \in R} \frac{\left|x_{u}(t)-x_{v}(t)\right|}{\sigma(t)} \rightarrow 0, u, v \rightarrow+\infty$, we know that $A_{u}^{-}$is a Cauchy sequence. Then $\lim _{u \rightarrow+\infty} A_{u}^{-}$exists.

By $\sup _{t \in R} \frac{\left|x_{u}(t)-x_{0}(t)\right|}{\sigma(t)} \rightarrow 0, u \rightarrow+\infty$, we get that

$$
\lim _{t \rightarrow-\infty} x_{0}(t)=\lim _{t \rightarrow-\infty} \lim _{u \rightarrow+\infty} \frac{x_{u}(t)}{\sigma(t)}=\lim _{u \rightarrow+\infty} \lim _{t \rightarrow-\infty} \frac{x_{u}(t)}{\sigma(t)}=\lim _{u \rightarrow+\infty} A_{u}^{-} .
$$

Hence $\lim _{t \rightarrow-\infty} x_{0}(t)$ exists. Similarly we can prove that $\lim _{t \rightarrow+\infty} x_{0}(t), \lim _{t \rightarrow-\infty} y_{0}(t)$, $\lim _{t \rightarrow+\infty} y_{0}(t)$ exist.

Step 3. Prove that $y_{0}(t)=\frac{\rho(t)\left[\sigma(t) x_{0}(t)\right]^{\prime}}{\Phi^{-1}(\tau(t))}$.

We have for $t>\xi$ and some $c_{u} \in \mathbb{R}$ that

$$
\begin{aligned}
& \left|x_{u}(t)-\sum_{\xi \leq t_{s}<t} \Delta x_{u}\left(t_{s}\right)-x_{u}(\xi)-\int_{\xi}^{t} \frac{\Phi^{-1}(\tau(s)) y_{0}(s)}{\rho(s)} d s\right| \\
& =\left|\int_{\xi}^{t} x_{u}^{\prime}(s) d s-\int_{\xi}^{t} \frac{\Phi^{-1}(\tau(s)) y_{0}(s)}{\rho(s)} d s\right| \\
& =\int_{\xi}^{t}\left|x_{u}^{\prime}(s)-\frac{\Phi^{-1}(\tau(s)) y_{0}(s)}{\rho(s)}\right| d s \leq \int_{\xi}^{t} \frac{\Phi^{-1}(\tau(s))}{\rho(s)} d s \sup _{t \in R}\left|\frac{\rho(t) x_{u}^{\prime}(t)}{\Phi^{-1}(\tau(t))}-y_{0}(t)\right| \\
& \leq \int_{\xi}^{t} \frac{\Phi^{-1}(\tau(s))}{\rho(s)} d s \sup _{t \in R}\left|\frac{\rho(t) x_{u}^{\prime}(t)}{\Phi^{-1}(\tau(t))}-y_{0}(t)\right| \rightarrow 0 \text { as } u \rightarrow+\infty .
\end{aligned}
$$

So

$$
\lim _{u \rightarrow+\infty}\left(x_{u}(t)-\sum_{\xi \leq t_{s}<t} \Delta x_{u}\left(t_{s}\right)-x_{u}(\xi)\right)=\int_{\xi}^{t} \frac{\Phi^{-1}(\tau(s)) y_{0}(s)}{\rho(s)} d s .
$$

Then

$$
\sigma(t) x_{0}(t)-\sum_{\xi \leq t_{s}<t} \Delta \sigma\left(t_{s}\right) x_{0}\left(t_{s}\right)-\sigma(\xi) c_{0}=\int_{\xi}^{t} \frac{\Phi^{-1}(\tau(s)) y_{0}(s)}{\rho(s)} d s .
$$

It follows that $\frac{\Phi^{-1}(\tau(s)) y_{0}(t)}{\rho(t)}=\left[\sigma(t) x_{0}(t)\right]$. That is $y_{0}(t)=\frac{\rho(t)\left[\sigma(t) x_{0}(t)\right]^{\prime}}{\Phi^{-1}(\tau(t))}$. Similarly we can get $y_{0}(t)=\frac{\rho(t)\left[\sigma(t) x_{0}(t)\right]^{\prime}}{\Phi^{-1}(\tau(t))}$ for $t<\xi$. So $x_{0} \in X$ with $x_{u} \rightarrow x_{0}$ as $u \rightarrow+\infty$. It follows that $X$ is a Banach space.

Lemma 2.2. Let $M$ be a subset of $X$. Then $M$ is relatively compact if and only if the following conditions are satisfied:

(i) both $\left\{t \rightarrow \frac{x(t)}{\sigma(t)}: x \in M\right\}$ and $\left\{t \rightarrow \frac{\rho(t) x^{\prime}(t)}{\Phi^{-1}(\tau(t))}: x \in M\right\}$ are uniformly bounded,

(ii) both $\left\{t \rightarrow \frac{x(t)}{\sigma(t)}: x \in M\right\}$ and $\left\{t \rightarrow \frac{\rho(t) x^{\prime}(t)}{\Phi^{-1}(\tau(t))}: x \in M\right\}$ are equicontinuous in $\left(t_{s}, t_{s+1}\right](s \in N)$,

(iii) both $\left\{t \rightarrow \frac{x(t)}{\sigma(t)}: x \in M\right\}$ and $\left\{t \rightarrow \frac{\rho(t) x^{\prime}(t)}{\Phi^{-1}(\tau(t))}: x \in M\right\}$ are equiconvergent as $t \rightarrow \pm \infty$. 
Proof. " $\Leftarrow$ ". From Lemma 2.1, we know $X$ is a Banach space. In order to prove that the subset $M$ is relatively compact in $X$, we only need to show $M$ is totally bounded in $X$, that is for all $\epsilon>0, M$ has a finite $\epsilon$-net.

For any given $\epsilon>0$, by (i)-(iii), there exist constants $M>0, \delta>0$, $t_{s_{0}}>0$ and $t_{-s_{0}}<0$ such that

$$
\begin{gathered}
\sup _{t \in R} \frac{|x(t)|}{\sigma(t)}, \sup _{t \in R} \frac{\rho(t)\left|x^{\prime}(t)\right|}{\Phi^{-1}(\tau(t))} \leq M, x \in M, \\
\left|\frac{x\left(w_{1}\right)}{\sigma\left(w_{1}\right)}-\frac{x\left(w_{2}\right)}{\sigma\left(w_{2}\right)}\right| \leq \frac{\epsilon}{3}, w_{1}, w_{2} \in\left(t_{s}, t_{s+1}\right],\left|w_{1}-w_{2}\right|<\delta, \\
s=-s_{0},-s_{0}+1, \cdots, s_{0}-1, x \in M, \\
\left|\frac{\rho\left(w_{1}\right) x^{\prime}\left(w_{1}\right)}{\Phi^{-1}\left(\tau\left(w_{1}\right)\right)}-\frac{\rho\left(w_{2}\right) x^{\prime}\left(w_{2}\right)}{\Phi^{-1}\left(\operatorname{tau}\left(w_{2}\right)\right)}\right|<\frac{\epsilon}{3}, \\
w_{1}, w_{2} \in\left(t_{s}, t_{s+1}\right],\left|w_{1}-w_{2}\right|<\delta, s=-s_{0},-s_{0}+1, \cdots, s_{0}-1, x \in M, \\
\left|\frac{x\left(w_{1}\right)}{\sigma\left(w_{1}\right)}-\frac{x\left(w_{2}\right)}{\sigma\left(w_{2}\right)}\right| \leq \frac{\epsilon}{3}, w_{1}, w_{2} \leq t_{-s_{0}} \text { or } w_{1}, w_{2} \geq t_{s_{0}}, x \in M, \\
\left|\frac{\rho\left(w_{1}\right) x^{\prime}\left(w_{1}\right)}{\Phi^{-1}\left(\tau\left(w_{1}\right)\right)}-\frac{\rho\left(w_{2}\right) x^{\prime}\left(w_{2}\right)}{\Phi^{-1}\left(\operatorname{tau}\left(w_{2}\right)\right)}\right|<\frac{\epsilon}{3}, w_{1}, w_{2} \leq t_{-s_{0}} \text { or } w_{1}, w_{2} \geq t_{s_{0}}, x \in M .
\end{gathered}
$$

Define

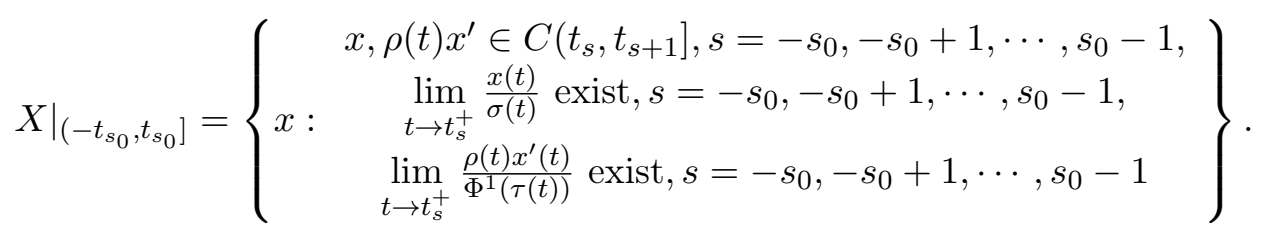

For $\left.x \in X\right|_{\left(-t_{s_{0}}, t_{s_{0}}\right]}$, define

$$
\|x\|_{s_{0}}=\max \left\{\max _{t \in\left(-t_{s_{0}}, t_{s_{0}}\right]} \frac{|x(t)|}{\sigma(t)}, \max _{t \in\left(-t_{s_{0}}, t_{s_{0}}\right]} \frac{\rho(t)\left|x^{\prime}(t)\right|}{\Phi^{-1}(\tau(t))}\right\} .
$$

Similarly to Lemma 2.1, we can prove that $X_{\left(-t_{s_{0}}, t_{s_{0}}\right]}$ is a Banach space.

Let $\left.M\right|_{\left(-t_{s_{0}}, t_{s_{0}}\right]}=\left\{t \rightarrow x(t), t \in\left(-t_{s_{0}}, t_{s_{0}}\right]: x \in M\right\}$. Then $\left.M\right|_{\left(-t_{s_{0}}, t_{s_{0}}\right]}$ is a subset of $\left.X\right|_{\left(-t_{s_{0}}, t_{s_{0}}\right]}$. By (i) and (ii), and Ascoli-Arzela theorem, we can know that $\left.M\right|_{\left(-t_{s_{0}}, t_{s_{0}}\right]}$ is relatively compact. Thus, there exist $x_{1}, x_{2}, \cdots, x_{k} \in M$ such that, for any $x \in M$, we have that there exists some $i=1,2, \cdots, k$ such that

$$
\left\|x-x_{i}\right\|_{s_{0}}=\max \left\{\sup _{t \in\left(-t_{s_{0}}, t_{s_{0}}\right]} \frac{\left|x(t)-x_{i}(t)\right|}{\sigma(t)}, \sup _{t \in\left(-t_{s_{0}}, t_{s_{0}}\right]} \frac{\rho(t)\left|x^{\prime}(t)-x_{i}^{\prime}(t)\right|}{\Phi^{-1}(\tau(t))}\right\}<\frac{\epsilon}{3} .
$$

Therefore, for $x \in M$, we have that

$$
\left\|x-x_{i}\right\|_{X}=\max \left\{\sup _{t \in\left(-t_{s_{0}}, t_{s_{0}}\right]} \frac{\left|x(t)-x_{i}(t)\right|}{\sigma(t)}, \sup _{t \in\left(-t_{s_{0}}, t_{s_{0}}\right]} \frac{\rho(t)\left|x^{\prime}(t)-x_{i}^{\prime}(t)\right|}{\Phi^{-1}(\tau(t))},\right.
$$




$$
\begin{aligned}
& \left.\sup _{t \geq t_{s_{0}}} \frac{\left|x(t)-x_{i}(t)\right|}{\sigma(t)}, \sup _{t \geq t_{s_{0}}} \frac{\rho(t)\left|x^{\prime}(t)-x_{i}^{\prime}(t)\right|}{\Phi^{-1}(\tau(t))} \sup _{t \leq t_{-s_{0}}} \frac{\left|x(t)-x_{i}(t)\right|}{\sigma(t)}, \sup _{t \leq t_{-s_{0}}} \frac{\rho(t)\left|x^{\prime}(t)-x_{i}^{\prime}(t)\right|}{\Phi^{-1}(\tau(t))}\right\} \\
& \leq \max \left\{\frac{\epsilon}{3}, \sup _{t \geq t_{s_{0}}}\left|\frac{x(t)}{\sigma(t)}-\frac{x\left(t_{s_{0}}^{+}\right)}{\sigma\left(t_{s_{0}}^{+}\right)}\right|+\sup _{t \geq t_{s_{0}}}\left|\frac{x\left(t_{s_{0}}^{+}\right)}{\sigma\left(t_{s_{0}}^{+}\right)}-\frac{x_{i}\left(t_{s_{0}}^{+}\right)}{\sigma\left(t_{s_{0}}^{+}\right)}\right|+\sup _{t \geq t_{s_{0}}^{+}}\left|\frac{x_{i}\left(t_{s_{0}}^{+}\right)}{\sigma\left(t_{s_{0}}^{+}\right)}-\frac{x_{i}(t)}{\sigma(t)}\right|,\right. \\
& \sup _{t \geq t_{s_{0}}}\left|\frac{\rho(t) x^{\prime}(t)}{\Phi 1(\tau(t))}-\frac{\rho\left(t_{s_{0}}^{+}\right) x^{\prime}\left(t_{s_{0}}^{+}\right)}{\Phi^{-1}\left(\tau\left(t_{s_{0}}^{+}\right)\right)}\right|+\sup _{t \geq t_{s_{0}}}\left|\frac{\rho\left(t_{s_{0}}^{+}\right) x^{\prime}\left(t_{s_{0}}^{+}\right)}{\Phi^{-1}\left(\tau\left(\left(t_{s_{0}}^{+}\right)\right)\right.}-\frac{\rho\left(t_{s_{0}}^{+}\right) x_{i}^{\prime}\left(t_{s_{0}}^{+}\right)}{\Phi^{-1}\left(\tau\left(\left(t_{s_{0}}^{+}\right)\right)\right.}\right| \\
& +\sup _{t \geq t_{s_{0}}}\left|\frac{\rho\left(t_{s_{0}}^{+}\right) x_{i}^{\prime}\left(t_{s_{0}}^{+}\right)}{\Phi^{-1}\left(\tau\left(\left(t_{s_{0}}^{+}\right)\right)\right.}-\frac{\rho(t) x_{i}^{\prime}(t)}{\Phi 1(\tau(t))}\right| \\
& \sup _{t \leq t_{-s_{0}}}\left|\frac{x(t)}{\sigma(t)}-\frac{x\left(t_{-s_{0}}\right)}{\sigma\left(t_{-s_{0}}\right)}\right|+\sup _{t \leq t_{-s_{0}}}\left|\frac{x\left(t_{-s_{0}}\right)}{\sigma\left(t_{-s_{0}}\right)}-\frac{x_{i}\left(t_{-s_{0}}\right)}{\sigma\left(t_{-s_{0}}\right)}\right|+\sup _{t \leq t_{-s_{0}}}\left|\frac{x_{i}\left(t_{-s_{0}}\right)}{\sigma\left(t_{-s_{0}}\right)}-\frac{x_{i}(t)}{\sigma(t)}\right|, \\
& \sup _{t \leq t_{-s_{0}}}\left|\frac{\rho(t) x^{\prime}(t)}{\Phi 1(\tau(t))}-\frac{\rho\left(t_{-s_{0}}\right) x^{\prime}\left(t_{-s_{0}}\right)}{\Phi^{-1}\left(\tau\left(t_{-s_{0}}\right)\right)}\right|+\sup _{t \leq t_{-s_{0}}}\left|\frac{\rho\left(t_{-s_{0}}\right) x^{\prime}\left(t_{-s_{0}}\right)}{\Phi^{-1}\left(\tau\left(t_{-s_{0}}\right)\right)}-\frac{\rho\left(t_{-s_{0}}\right) x_{i}^{\prime}\left(t_{-s_{0}}\right)}{\Phi^{-1}\left(\tau\left(t_{-s_{0}}\right)\right)}\right| \\
& \left.+\sup _{t \leq t_{-s_{0}}}\left|\frac{\rho\left(t_{-s_{0}}\right) x_{i}^{\prime}\left(t_{-s_{0}}\right)}{\Phi^{-1}\left(\tau\left(t_{-s_{0}}\right)\right)}-\frac{\rho(t) x_{i}^{\prime}(t)}{\Phi^{-1}(\tau(t))}\right|\right\} \leq \epsilon .
\end{aligned}
$$

So, for any $\epsilon>0, M$ has a finite $\epsilon$-net $\left\{U_{x_{1}}, U_{x_{2}}, \cdots, U_{x_{k}}\right\}$, that is, $M$ is totally bounded in $X$. Hence $M$ is relatively compact in $X$.

$\Rightarrow$. Assume that $M$ is relatively compact, then for any $\epsilon>0$, there exists a finite $\epsilon$-net of $M$. Let the finite $\epsilon$-net be $\left\{U_{x_{1}}, U_{x_{2}}, \cdots, U_{x_{k}}\right\}$ with $x_{i} \subset M$. Then for any $x \in M$, there exists $U_{x_{i}}$ such that $x \in U_{x_{i}}$ and $\|x\| \leq\left\|x-x_{i}\right\|+\left\|x_{i}\right\| \leq \epsilon+\max \left\{\left\|x_{i}\right\|: i=1,2, \cdots, k\right\}$. It follows that both $\left\{t \rightarrow \frac{x(t)}{\sigma(t)}: x \in M\right\}$ and $\left\{t \rightarrow \frac{\rho(t) x^{\prime}(t)}{\Phi^{-1}(\tau(t))}: x \in M\right\}$ are uniformly bounded. Then (i) holds.

Furthermore, there exists $t_{-s_{0}}<0$ and $t_{s_{0}}>0$ such that $\mid x_{i}\left(w_{1}\right)-$ $x_{i}\left(w_{2}\right) \mid<\epsilon$ for all $w_{1}, w_{2} \geq t_{s_{0}}$ and all $w_{1}, w_{2} \leq t_{-s_{0}}$ and $i=1,2, \cdots, k$. Then we have for $w_{1}, w_{2} \geq t_{s_{0}}$ and all $w_{1}, w_{2} \leq t_{-s_{0}}$ that

$$
\begin{aligned}
& \left|\frac{x\left(w_{1}\right)}{\sigma\left(w_{1}\right)}-\frac{x\left(w_{2}\right)}{\sigma\left(w_{2}\right)}\right| \\
& \leq\left|\frac{x\left(w_{1}\right)}{\sigma\left(w_{1}\right)}-\frac{x_{i}\left(w_{1}\right)}{\sigma\left(w_{2}\right)}\right|+\left|\frac{x_{i}\left(w_{1}\right)}{\sigma\left(w_{1}\right)}-\frac{x_{i}\left(w_{2}\right)}{\sigma\left(w_{2}\right)}\right|+\left|\frac{x_{i}\left(w_{2}\right)}{\sigma\left(w_{2}\right)}-\frac{x\left(w_{2}\right)}{\sigma\left(w_{2}\right)}\right|<3 \epsilon, x \in M .
\end{aligned}
$$

Similarly we have for $w_{1}, w_{2} \geq t_{s_{0}}$ and all $w_{1}, w_{2} \leq t_{-s_{0}}$ that

$$
\left|\frac{\rho\left(w_{1}\right) x^{\prime}\left(w_{1}\right)}{\Phi^{-1}\left(\tau\left(w_{1}\right)\right)}-\frac{\rho\left(w_{2}\right) x^{\prime}\left(w_{2}\right)}{\Phi^{-1}\left(\tau\left(w_{2}\right)\right)}\right|<3 \epsilon, x \in M .
$$

Thus (iii) is valid. Similarly we can prove that (ii) holds. Consequently, the Lemma is proved. 
For ease expression, we define $\sum_{a \leq t_{s}<b} k_{s}:=-\sum_{b \leq t_{s}<a} k_{s}$ for $a>b$. For $x \in X$, define $(T x)(t)$ by

$$
\begin{aligned}
& (T x)(t)=\int_{-\infty}^{+\infty} m(s) \phi\left(s, x(s), \rho(s) x^{\prime}(s)\right) d s+\sum_{\xi \leq t_{s}<t} I\left(t_{s}, x\left(t_{s}\right), \rho\left(t_{s}\right) x^{\prime}\left(t_{s}\right)\right) \\
& +\int_{\xi}^{t} \frac{1}{\rho(u)} \Phi^{-1}\left(\Phi\left(\int_{-\infty}^{+\infty} n(s) \psi\left(s, x(s), \rho(s) x^{\prime}(s)\right) d s\right)\right. \\
& \left.+\sum_{\eta \leq t_{s}<u} J\left(t_{s}, x\left(t_{s}\right), \rho\left(t_{s}\right) x^{\prime}\left(t_{s}\right)\right)-\int_{\eta}^{u} p(w) f\left(w, x(w), \rho(w) x^{\prime}(w)\right) d w\right) d u .
\end{aligned}
$$

Lemma 2.3. Suppose that $f, \phi, \psi$ are weak Carathéodory functions and $I, J$ are discrete Carathéodory functions and for each $r>0, f\left(t, \sigma(t) u, \Phi^{-1}(\tau(t)) v\right)$ converges uniformly as $t \rightarrow \pm \infty$ on $[-r, r] \times[-r, r]$. Then $T: X \rightarrow X$ is well defined and is completely continuous, $x \in X$ is a solution of (1.1) if and only if $x \in X$ is a fixed point of $T$ in $X$.

Proof. From Lemma 2.1, $X$ is a Banach space. For $x \in X$, we have $\|x\| \leq r$ for some $r \geq 0$. Then there exists constants $M_{r, f} \geq 0, M_{r, J, s} \geq 0$ and $M_{r, I, s} \geq 0$ such that

$$
\begin{aligned}
& \left|f\left(t, x(t), x^{\prime}(t)\right)\right|=\left|f\left(t, \sigma(t) \frac{x(t)}{\sigma(t)}, \Phi^{-1}(\tau(t)) \frac{\rho(t) x^{\prime}(t)}{\Phi^{-1}(\tau(t))}\right)\right| \leq M_{r, f}, \text { a.e., } t \in R, \\
& \left|\phi\left(t, x(t), \rho(t) x^{\prime}(t)\right)\right| \leq M_{r, \phi}, \text { a.e., } t \in R, \\
& \left|\psi\left(t, x(t), \rho(t) x^{\prime}(t)\right)\right| \leq M_{r, \psi}, \text { a.e., } t \in R, \\
& \quad\left|I\left(t_{s}, x\left(t_{s}\right), \rho\left(t_{s}\right) x^{\prime}\left(t_{s}\right)\right)\right| \leq M_{r, I, s}, s \in Z, \sum_{s=-\infty}^{+\infty} M_{r, I, s}<+\infty, \\
& \left|J\left(t_{s}, x\left(t_{s}\right), \rho\left(t_{s}\right) x^{\prime}\left(t_{s}\right)\right)\right| \leq M_{r, J, s}, s \in Z, \sum_{s=-\infty}^{+\infty} M_{r, J, s}<+\infty .
\end{aligned}
$$

By direct computation, we get

$$
\left\{\begin{array}{l}
{\left[\Phi\left(\rho(t)(T x)^{\prime}(t)\right)\right]^{\prime}+p(t) f\left(t, x(t), \rho(t) x^{\prime}(t)\right)=0, \quad \text { a.e., } t \in \mathbb{R},} \\
(T x)(\xi)=\int_{-\infty}^{+\infty} m(s) \phi\left(s, x(s), \rho(s) x^{\prime}(s)\right) d s, \\
\rho(\eta)(T x)^{\prime}(\eta)=\int_{-\infty}^{+\infty} n(s) \psi\left(s, x(s), \rho(s) x^{\prime}(s)\right) d s, \\
\Delta(T x)\left(t_{i}\right)=I\left(t_{i}, x\left(t_{i}\right), \rho\left(t_{i}\right) x^{\prime}\left(t_{i}\right)\right), i \in \mathbb{Z}, \\
\Delta \Phi\left(\rho\left(t_{i}\right)(T x)^{\prime}\left(t_{i}\right)\right)=J\left(t_{i}, x\left(t_{i}\right), \rho\left(t_{i}\right) x^{\prime}\left(t_{i}\right)\right), i \in \mathbb{Z} .
\end{array}\right.
$$


One can show that $T x \in X$. Thus $T: X \rightarrow X$ is well defined and $x \in X$ is a solution of (1) if and only if $x \in X$ is a fixed point of $T$ in $X$. Similarly to the proof of Lemma 2.2 in [18], we can prove that $T$ is completely continuous. The proof is complete.

Lemma 2.4. Let $X$ be a Banach space. Assume that $\Omega$ is an open bounded subset of $X$ with $0 \in \Omega$ and let $T: X \rightarrow X$ be a completely continuous operator such that $\|T x\| \leq\|x\|$ for all $x \in \partial \Omega$. Then $T$ has a fixed point in $\Omega$.

\section{MAin RESUlt}

In this section, we prove the main result of this paper. We need the following assumption:

(A) there exist nonnegative constants $A_{j}, a_{i j}, b_{i j} \geq 0(i=1,2, j=$ $0,1,2, \cdots, m), \phi_{s} \geq 0, \psi_{s} \geq 0(s \in \mathbb{Z})$ and $k_{j}, l_{j} \geq 0(j=1,2, \cdots, m)$ with $k_{j}+l_{j}>0$ and $\sum_{s=-\infty}^{+\infty} \phi_{s}<+\infty, \quad \sum_{s=-\infty}^{+\infty} \psi_{s}<+\infty$ and

$$
\begin{aligned}
& \left|f\left(t, \sigma(t) u, \Phi^{-1}(\tau(t)) v\right)\right| \leq \Phi\left(A_{0}+\sum_{j=1}^{m} A_{j}|u|^{k_{j}}|v|^{l_{j}}\right), \\
& \left|I\left(t_{s}, \sigma\left(t_{s}\right) u, \Phi^{-1}\left(\tau\left(t_{s}\right)\right) v\right)\right| \leq \phi_{s}\left[b_{10}+\sum_{j=1}^{m} b_{1 j}|u|^{k_{j}}|v|^{l_{j}}\right], \\
& \left|J\left(t_{s}, \sigma\left(t_{s}\right) u, \Phi^{-1}\left(\tau\left(t_{s}\right)\right) v\right)\right| \leq \psi_{s} \Phi\left(b_{20}+\sum_{j=1}^{m} b_{2 j}|u|^{k_{j}}|v|^{l_{j}}\right), \\
& \left|\phi\left(t, \sigma(t) u, \Phi^{-1}(\tau(t)) v\right)\right| \leq a_{10}+\sum_{j=1}^{m} a_{1 j}|u|^{k_{j}}|v|^{l_{j}}, \\
& \left|\psi\left(t, \sigma(t) u, \Phi^{-1}(\tau(t)) v\right)\right| \leq a_{20}+\sum_{j=1}^{m} a_{2 j}|u|^{k_{j}}|v|^{l_{j}},
\end{aligned}
$$

hold for all $u, v \in R$, a.e.t $\in \mathbb{R}, s \in \mathbb{Z}$.

We denote

$$
\begin{aligned}
& \sigma=\max \left\{k_{j}+l_{j}: j=1,2, \cdots, m\right\}, \\
& \bar{A}_{1}=\|m\|_{1} a_{10}+\sum_{s=-\infty}^{+\infty} \phi_{s} b_{1,0}+c_{l}\|n\|_{1} a_{20}+c_{l} \Phi^{-1}\left(\sum_{s=-\infty}^{+\infty} \psi_{s}\right) b_{2,0}+c_{l} A_{0}, \\
& \bar{B}_{1, j}=\|m\|_{1} a_{1 j}+\sum_{s=-\infty}^{+\infty} \phi_{s} b_{1, j}+c_{l}\|n\|_{1} a_{2 j}+c_{l} \Phi^{-1}\left(\sum_{s=-\infty}^{+\infty} \psi_{s}\right) b_{2, j}+c_{l} A_{j},
\end{aligned}
$$


and

$$
\begin{aligned}
& \bar{A}_{2}=c_{l}\|n\|_{1} a_{10}+c_{l} \Phi^{-1}\left(\sum_{s=-\infty}^{+\infty} \psi_{s}\right) b_{2,0}+c_{l} A_{0}, \\
& \bar{B}_{2, j}=c_{l}\|n\|_{1} a_{1 j}+c_{l} \Phi^{-1}\left(\sum_{s=-\infty}^{+\infty} \psi_{s}\right) b_{2 j}+c_{l} A_{j}, \\
& A=\max \left\{\bar{A}_{1}, \bar{A}_{2}\right\}, \quad B=\max \left\{\sum_{j=1}^{m} \bar{B}_{1, j}, \sum_{j=1}^{m} \bar{B}_{2, j}\right\} .
\end{aligned}
$$

Theorem 3.1. Suppose that (A) holds, $f, \phi, \psi$ are weak Carathéodory functions and $I, J$ are discrete Carathéodory functions and for each $r>0$, $f\left(t, \sigma(t) u, \Phi^{-1}(\tau(t)) v\right)$ converges uniformly as $t \rightarrow \pm \infty$ on $[-r, r] \times[-r, r]$. Then $B V P(1)$ has at least one solution if

(i) $\sigma \in(0,1)$ or (ii) $\sigma=1$ and $B<1$ or (iii) $\sigma>1$ and $B(A+B)^{\sigma-1} \leq$ $\frac{(\sigma-1)^{\sigma-1}}{\sigma^{\sigma}}$.

Proof. We will apply Lemma 2.4. Let $X$ and $T$ be defined in Section 2 . From Lemma 2.3, $T: X \rightarrow X$ is well defined and is a completely continuous operator. We prove that $T$ has a fixed point in $X$ to get a solution of $\operatorname{BVP}(1)$. For $x \in X$, we have $\|x\| \leq r<+\infty$. Then (A) implies that

$$
\begin{aligned}
& \left|f\left(t, x(t), \rho(t) x^{\prime}(t)\right)\right|=\left|f\left(t, \sigma(t) \frac{x(t)}{\sigma(t)}, \Phi^{-1}(\tau(t)) \frac{\rho(t) x^{\prime}(t)}{\Phi^{-1}(\tau(t))}\right)\right| \\
& \leq \Phi\left(A_{0}+\sum_{j=1}^{m} A_{j}\left|\frac{x(t)}{\sigma(t)}\right|^{k_{j}}\left|\frac{\rho(t) x^{\prime}(t)}{\Phi^{-1}(\tau(t))}\right|^{l_{j}}\right) \leq \Phi\left(A_{0}+\sum_{j=1}^{m} A_{j} r^{k_{j}+l_{j}}\right), t \in \mathbb{R}, \\
& \left|I\left(t_{s}, x\left(t_{s}\right), \rho\left(t_{s}\right) x^{\prime}\left(t_{s}\right)\right)\right| \leq \phi_{s}\left[b_{10}+\sum_{j=1}^{m} b_{1 j} r^{k_{j}+l_{j}}\right], s \in \mathbb{Z}, \\
& \left|J\left(t_{s}, x\left(t_{s}\right), \rho\left(t_{s}\right) x^{\prime}\left(t_{s}\right)\right)\right| \leq \psi_{s} \Phi\left(b_{20}+\sum_{j=1}^{m} b_{2 j} r^{k_{j}+l_{j}}\right), s \in \mathbb{Z}, \\
& \left|\phi\left(t, x(t), \rho(t) x^{\prime}(t)\right)\right| \leq a_{10}+\sum_{j=1}^{m} a_{1 j} r^{k_{j}+l_{j}}, \text { a.e., } t \in \mathbb{R}, \\
& \left|\psi\left(t, x(t), \rho(t) x^{\prime}(t)\right)\right| \leq a_{20}+\sum_{j=1}^{m} a_{2 j} r^{k_{j}+l_{j}}, \text { a.e. }, t \in \mathbb{R} .
\end{aligned}
$$


By the definition of $T$, we get

$$
\begin{aligned}
& \frac{|(T x)(t)|}{\sigma(t)} \leq \frac{1}{1+\left|\int_{\xi}^{t} \frac{\Phi^{-1}(\tau(u))}{\rho(u)} d u\right|}\left[\int_{-\infty}^{+\infty}|m(s)|\left|\phi\left(s, x(s), \rho(s) x^{\prime}(s)\right)\right| d s\right. \\
& +\left|\sum_{\xi \leq t_{s}<t}\right| I\left(t_{s}, x\left(t_{s}\right), \rho\left(t_{s}\right) x^{\prime}\left(t_{s}\right)\right)|| \\
& +\mid \int_{\xi}^{t} \frac{1}{\rho(u)} \Phi^{-1}\left(\Phi\left(\int_{-\infty}^{+\infty}|n(s)|\left|\psi\left(s, x(s), \rho(s) x^{\prime}(s)\right)\right| d s\right)\right. \\
& +\sum_{\eta \leq t_{s}<u}\left|J\left(t_{s}, x\left(t_{s}\right), \rho\left(t_{s}\right) x^{\prime}\left(t_{s}\right)\right)\right| \mid \\
& \left.\left.+\left|\int_{\eta}^{u} p(w)\right| f\left(w, x(w), \rho(w) x^{\prime}(w)\right)|d w|\right) d u \mid\right] \\
& \leq\|m\|_{1}\left[a_{10}+\sum_{j=1}^{m} a_{1 j} r^{k_{j}+l_{j}}\right]+\sum_{s=-\infty}^{+\infty} \phi_{s}\left[b_{10}+\sum_{j=1}^{m} b_{1 j} r^{k_{j}+l_{j}}\right] \\
& +\frac{\left|\int_{\xi}^{t} \frac{\Phi^{-1}(\tau(u))}{\rho(u)} d u\right|}{1+\left|\int_{\xi}^{t} \frac{\Phi^{-1}(\tau(u))}{\rho(u)} d u\right|} \Phi^{-1}\left(\Phi\left(\|n\|_{1}\left(a_{20}+\sum_{j=1}^{m} a_{2 j} r^{k_{j}+l_{j}}\right)\right)\right. \\
& \left.+\Phi\left(b_{20}+\sum_{j=1}^{m} b_{2 j} r^{k_{j}+l_{j}}\right) \sum_{s=-\infty}^{+\infty} \psi_{s}+\Phi\left(A_{0}+\sum_{j=1}^{m} A_{j} r^{k_{j}+l_{j}}\right)\right) .
\end{aligned}
$$

One knows that

$$
\Phi^{-1}(u+v) \leq c_{l}\left[\Phi^{-1}(u)+\Phi^{-1}(v)\right], u, v \geq 0 \text { with } c_{l}=\left\{\begin{array}{l}
1,1<l<2 \\
2^{l-1}, l \geq 2
\end{array}\right.
$$

It follows that

$$
\begin{aligned}
& \sup _{t \in \mathbb{R}} \frac{|(T x)(t)|}{\sigma(t)} \leq\|m\|_{1}\left[a_{10}+\sum_{j=1}^{m} a_{1 j} r^{k_{j}+l_{j}}\right]+\sum_{s=-\infty}^{+\infty} \phi_{s}\left[b_{1,0}+\sum_{j=1}^{m} b_{1, j} r^{k_{j}+l_{j}}\right] \\
& +c_{l}\left(\|n\|_{1}\left(a_{20}+\sum_{j=1}^{m} a_{2 j} r^{k_{j}+l_{j}}\right)\right) \\
& +c_{l}\left(b_{20}+\sum_{j=1}^{m} b_{2 j} r^{k_{j}+l_{j}}\right) \Phi^{-1}\left(\sum_{s=-\infty}^{+\infty} \psi_{s}\right)+c_{l}\left(A_{0}+\sum_{j=1}^{m} A_{j} r^{k_{j}+l_{j}}\right)
\end{aligned}
$$




$$
\begin{aligned}
& =\|m\|_{1} a_{10}+\sum_{s=-\infty}^{+\infty} \phi_{s} b_{1,0}+c_{l}\|n\|_{1} a_{20}+c_{l} \Phi^{-1}\left(\sum_{s=-\infty}^{+\infty} \psi_{s}\right) b_{2,0}+c_{l} A_{0} \\
& +\sum_{j=1}^{m}\left(\|m\|_{1} a_{1 j}+\sum_{s=-\infty}^{+\infty} \phi_{s} b_{1, j}\right. \\
& \left.+c_{l}\|n\|_{1} a_{2 j}+c_{l} \Phi^{-1}\left(\sum_{s=-\infty}^{+\infty} \psi_{s}\right) b_{2, j}+c_{l} A_{j}\right) r^{k_{j}+l_{j}} .
\end{aligned}
$$

Then

$$
\sup _{t \in \mathbb{R}} \frac{|(T x)(t)|}{\sigma(t)} \leq \bar{A}_{1}+\sum_{j=1}^{m} \bar{B}_{1, j} r^{k_{j}+l_{j}} .
$$

On the other hand, we have

$$
\begin{aligned}
& \frac{\rho(t)\left|(T x)^{\prime}(t)\right|}{\Phi^{-1}(\tau(t))} \leq \frac{1}{\Phi^{-1}(\tau(t))} \mid \Phi^{-1}\left(\Phi\left(\|n\|_{1}\left(a_{10}+\sum_{j=1}^{m} a_{1 j} r^{k_{j}+l_{j}}\right)\right)\right. \\
& +\sum_{s=-\infty}^{+\infty} \psi_{s} \Phi\left(b_{20}+\sum_{j=1}^{m} b_{2 j} r^{k_{j}+l_{j}}\right) \\
& \left.+\left|\int_{\eta}^{t} p(w) d w\right| \Phi\left(A_{0}+\sum_{j=1}^{m} A_{j} r^{k_{j}+l_{j}}\right)\right) \mid \\
& \leq \Phi^{-1}\left(\Phi\left(\|n\|_{1}\left(a_{10}+\sum_{j=1}^{m} a_{1 j} r^{k_{j}+l_{j}}\right)\right)\right. \\
& \left.+\sum_{s=-\infty}^{+\infty} \psi_{s} \Phi\left(b_{20}+\sum_{j=1}^{m} b_{2 j} r^{k_{j}+l_{j}}\right)+\Phi\left(A_{0}+\sum_{j=1}^{m} A_{j} r^{k_{j}+l_{j}}\right)\right) \\
& +c_{l} \Phi^{-1}\left(\sum_{s=-\infty}^{+\infty} \psi_{s}\right)\left(b_{20}+\sum_{j=1}^{m} b_{2 j} r^{k_{j}+l_{j}}\right)+c_{l}\left(A_{0}+\sum_{j=1}^{m} A_{j} r^{k_{j}+l_{j}}\right) \\
& \leq c_{l}\left(\|n\|_{1}\left(a_{10}+\sum_{j=1}^{m} a_{1 j} r^{k_{j}+l_{j}}\right)\right)
\end{aligned}
$$

It follows that

$$
\sup _{t \in \mathbb{R}} \frac{\rho(t)\left|(T x)^{\prime}(t)\right|}{\Phi^{-1}(\tau(t))} \leq \bar{A}_{2}+\sum_{j=1}^{m} \bar{B}_{2, j}\|x\|^{k_{j}+l_{j}} .
$$

It follows from (3) and (4) that

$$
\|T x\| \leq A+B \max \left\{\|(x, y)\|^{\sigma}, 1\right\} \leq A+B+B\|x\|^{\sigma} .
$$


(i) $\sigma \in(0,1)$.

Since $\sigma \in(0,1)$, change $r_{0}>0$ such that $A+B+B r_{0}^{\sigma} \leq r_{0}$. Let $\Omega_{0}=\left\{x \in X:\|x\| \leq r_{0}\right\}$. Then we get $\|T x\| \leq A+B+B r_{0}^{\sigma} \leq r_{0}$. So $T \overline{\Omega_{0}} \subset \overline{\Omega_{0}}$. Then $\|T x\| \leq\|x\|$ for all $x \in \partial \Omega$. Thus Lemma 2.4 implies that the operator $T$ has at least one fixed point in $\overline{\Omega_{0}}$. So BVP $(1)$ has at least one solution.

(ii) $\sigma=1$.

Let $r_{0}=\frac{A+B}{1-B}$ such that $A+B+B r_{0}=r_{0}$. Let $\Omega_{0}=\left\{x \in X:\|x\| \leq r_{0}\right\}$. Then we get $\|T x\| \leq A+B+B r_{0}^{\sigma} \leq r_{0}$. So $T \overline{\Omega_{0}} \subset \overline{\Omega_{0}}$. Then $\|T x\| \leq\|x\|$ for all $x \in \partial \Omega$. Thus Lemma 2.4 implies that the operator $T$ has at least one fixed point in $\overline{\Omega_{0}}$. So BVP(1) has at least one solution.

(iii) $\sigma>1$.

Let $r_{0}=\left(\frac{A+B}{B(\sigma-1)}\right)^{\frac{1}{\sigma}}$. It is easy to show from $\frac{(A+B)^{\sigma-1} \sigma^{\sigma}}{(\sigma-1)^{\sigma-1}} \leq \frac{1}{B}$ that $A+B+B r_{0}^{\sigma} \leq r_{0}$. Let $\Omega_{0}=\left\{x \in X:\|x\| \leq r_{0}\right\}$. Then we get $\|T x\| \leq$ $A+B+B r_{0}^{\sigma} \leq r_{0}$. So $T \overline{\Omega_{0}} \subset \overline{\Omega_{0}}$. Then $\|T x\| \leq\|x\|$ for all $x \in \partial \Omega$. Thus Lemma 2.4 implies that the operator $T$ has at least one fixed point in $\overline{\Omega_{0}}$. So BVP(1) has at least one solution.

The proof of Theorem 3.1 is completed.

\section{An EXAMPle}

In this section, we present an example to illustrate the main result.

Example 4.1. We consider the following BVP

$$
\left\{\begin{array}{l}
{\left[t\left|x^{\prime}(t)\right| x^{\prime}(t)\right]^{\prime}+f\left(t, x(t), \sqrt{|t|} x^{\prime}(t)\right)=0, \text { a.e. } t \in \mathbb{R},} \\
x(0)=\sqrt{\pi}, x^{\prime}(0)=2 \sqrt{\pi}, \\
\Delta x(s)=\lim _{t \rightarrow s^{+}} x(t)-x(s)=2^{-|s|}, s \in \mathbb{Z}, \\
\Delta\left(\sqrt{|s|} x^{\prime}(s)\right)^{3}=\lim _{t \rightarrow s^{+}}\left(\sqrt{|t|} x^{\prime}(t)\right)^{3}-\left(\sqrt{|s|} x^{\prime}(s)\right)^{3}=2^{-|s|}, s \in \mathbb{Z},
\end{array}\right.
$$

where

$$
f(t, u, v)=\frac{1}{1+|t|}\left(A_{0}+\sum_{j=1}^{m} A_{j}\left|\frac{u}{1+\sqrt{t^{2}+|t|}-\ln (\sqrt{1+|t|}-\sqrt{|t|})}\right|^{k_{j}}\left|\frac{v}{\sqrt{1+|t|}}\right|^{l_{j}}\right)^{2},
$$

$A_{j} \geq 0, k_{j}, l_{j} \geq 0(j=0,1,2, \cdots, m)$.

Corresponding to $\operatorname{BVP}(1)$, we have $\Phi(x)=|s| s$ and $\Phi^{-1}(x)=|s|^{-\frac{1}{2}} s$, $\rho(t)=\sqrt{|t|}, p(t)=1, \xi=\eta=0, t_{s}=s, s \in \mathbb{Z}, \phi(t, u, v)=1$ and 
$\psi(t, u, v)=2, m(t)=n(t)=e^{-t^{2}}, I(s, u, v)=J(s, u, v)=2^{-|s|}(s \in Z)$. One sees that

(i) $\Phi(x)=|x|^{k-2} x$ with $k=3>1$, the inverse of $\Phi$ is denoted by $\Phi^{-1}$ and $\Phi^{-1}(x)=|x|^{l-2} x$ with $l=\frac{3}{2}$,

(ii) $p: R \rightarrow[0, \infty)$ with $p \in L_{l o c}^{1}(R)$ and $\int_{-\infty}^{0} p(t) d t=\int_{0}^{+\infty} p(s) d s=$ $+\infty$, we find that $\tau(t)=1+|t|+\frac{t^{2}}{2}$,

(iii) one sees that $\rho: R \rightarrow[0, \infty)$ and

$$
\begin{aligned}
& \tau(t)=1+\left|\int_{0}^{t} p(s) d s\right|=1+|t|, \\
& \sigma(t)=1+\left|\int_{0}^{t} \frac{\Phi^{-1}(\tau(u))}{\rho(u)} d u\right| \\
& =1+\left|\int_{0}^{t} \frac{\sqrt{1+|u|}}{\sqrt{|u|}} d u\right|=1+\sqrt{t^{2}+|t|}-\ln (\sqrt{1+|t|}-\sqrt{|t|}) .
\end{aligned}
$$

with $\frac{\Phi^{-1}(\tau(\cdot))}{\rho(\cdot)} \in L_{l o c}^{1}(R)$ and $\int_{-\infty}^{0} \frac{\Phi^{-1}(\tau(u))}{\rho(u)} d u=\int_{0}^{+\infty} \frac{\Phi^{-1}(\tau(u))}{\rho(u)} d u=+\infty$, and $f, \phi, \psi$ are weak Carathéodory functions and $I, J$ are discrete Carathéodory functions and for each $r>0$,

$$
f\left(t, \sigma(t) u, \Phi^{-1}(\tau(t)) v\right)=\frac{1}{1+|t|}\left(A_{0}+\sum_{j=1}^{m} A_{j}|u|^{k_{j}}|v|^{l_{j}}\right)^{2} \rightarrow 0
$$

uniformly as $t \rightarrow \pm \infty$ on $[-r, r] \times[-r, r]$.

(iv) $f, \phi, \psi: \mathbb{R}^{3} \rightarrow \mathbb{R}$ are strong Carathéodory functions, $m, n \in L^{1}(\mathbb{R})$,

(v) $\left\{t_{s}: s \in \mathbb{Z}\right\}$ is a increasing sequence with $\lim _{s \rightarrow-\infty} t_{s}=-\infty$ and $\lim _{s \rightarrow+\infty} t_{s}=+\infty$

(vi) $I, J:\left\{t_{s}: s \in Z\right\} \times \mathbb{R}^{2} \rightarrow \mathbb{R}$ are discrete Carathéodory functions.

Choose $A_{j}(j=0,1,2, \cdots, m)$ and

$$
\begin{aligned}
& b_{1,0}=b_{2,0}=1, \quad b_{1, j}=b_{2, j}=0, j=1,2, \cdots, m, \\
& a_{1,0}=1, a_{2,0}=2, \quad a_{1, j}=a_{2, j}=0, j=1,2, \cdots, m .
\end{aligned}
$$

Then (A) holds and $\sum_{s=-\infty}^{+\infty} \phi_{s}=\sum_{s=-\infty}^{+\infty} \psi_{s}=3$. Denote $\sigma=\max \left\{k_{j}+l_{j}: j=\right.$ $1,2, \cdots, m\}$ and by direct computation, we get

$$
\begin{aligned}
& \bar{A}_{1}=3+\sqrt{3}+3 \sqrt{\pi}+A_{0}, \quad \bar{B}_{1, j}=A_{j}, \\
& \bar{A}_{2}=\sqrt{3}+\sqrt{\pi}+A_{0}, \quad \bar{B}_{2, j}=A_{j},
\end{aligned}
$$




$$
\begin{aligned}
& A=\max \left\{\bar{A}_{1}, \bar{A}_{2}\right\}=3+\sqrt{3}+3 \sqrt{\pi}+A_{0}, \\
& B=\max \left\{\sum_{j=1}^{m} \bar{B}_{1, j}, \sum_{j=1}^{m} \bar{B}_{2, j}\right\}=\sum_{j=1}^{m} A_{j} .
\end{aligned}
$$

By Theorem 3.1, BVP(5) has at least one solution if

(i) $\sigma \in(0,1)$ or

(ii) $\sigma=1$ and $\sum_{j=1}^{m} A_{j}<1$ or

(iii) $\sigma>1$ and $\sum_{j=1}^{m} A_{j}\left(3+\sqrt{3}+3 \sqrt{\pi}+A_{0}+\sum_{j=1}^{m} A_{j}\right)^{\sigma-1} \leq \frac{(\sigma-1)^{\sigma-1}}{\sigma^{\sigma}}$.

\section{REFERENCES}

[1] M. Arias, Fast and heteroclinic solutions for a second order ODE, Electronic Journal of Differential Equations, Conference 14, 2006, pp. 119-124.

[2] R. P. Agarwal, D. O'Regan, Infinite Interval Problems for Differential, Difference and Integral Equations . Kluwer Academic Publisher, Netherlands (2001).

[3] C. Avramescu, C. Vladimirescu, Existence of Homoclinic solutions to a nonlinear second order ODE, Dynamics of continuous, discrete and impulsive systems, Ser. A, Math Anal. 15(2008) 481-491.

[4] C. Avramescu, C. Vladimirescu, Existence of solutions to second order ordinary differential equations having finite limits at $\pm \infty$, Electronic Journal of differential equations, 18(2004) 1-12.

[5] C. Avramescu and C. Vladimirescu, Limits of solutions of a perturbed linear differential equation, E. J. Qualitative Theory of Diff. Equ., 3(2002) 1-11.

[6] D.G. Aronson and H.F. Weinberger, Nonlinear diffusion in population genetics, commbustion, and nerve propagation, in Partial Differential Equations and Related Topics, Lecture Notes in Mathematics, Vol. 446, pp. 5-49, Springer, New York, 1975.

[7] I. Bachar, H. Maagli, Existence and uniqueness for superlinear second-order differential equations on the half-line, Electron. J. Diff. Equ., Vol. 2015 (2015), No. 08, pp. $1-14$.

[8] A. A. Boichuk, O. A. Pokutnyi, Dichotomy and boundary value problems on the whole line, Chaotic Modeling and Simulation (CMSIM) 2 (2013) 247-255.

[9] B. Bianconi, F. Papalini, Non-autonomous boundary value problems on the real line, Discrete and continuous dynamical systems, 15(2006) 759-776.

[10] A. Calamai, Heteroclinic solutions of boundary value problems on the real line involving singular $\Phi$-Laplacian operators, Journal of Mathematical Analysis and Applications, 378(2011) 667-679.

[11] A. Cabada, J.A. Cid, Heteroclinic solutions for non-autonomous boundary value problems with singular $\Phi$-Laplacian operators, Discrete Contin. Dyn. Syst. 2009, Dynamical Systems, Differential Equations and Applications. 7th AIMS Conference, suppl., $118-122$. 
[12] G. Cupini, C. Marcelli, F. Papalini, Heteroclinic solutions of boundary value problems on the real line involving general nonlinear differential operators, Differ. Integral Equ. 24(7-8) (2011), 619-644.

[13] G. Cupini, C. Marcelli, F. Papalini, On the solvability of a boundary value problem on the real line, Bound. Value Probl. 2011, 26 (2011).

[14] F. Y. Deren, N. A. Hamal, Second-order boundary-value problems with integral boundary conditions on the real line, Electronic Journal of Differential Equations, 19 (2014) $1-13$.

[15] V. A. Il'in, E. I. Moiseev, Nonlocal boundary-value problem of the second kind for a Sturm-Liouville operator, Differential Equations 23 (1987) 979-987.

[16] Y. Liu, Multiple positive solutions to mixed boundary value problems for singular ordinary differential equations on the whole line, Nonlinear Analysis: Modelling and Control, 17 (4)(2012) 460-480.

[17] Y. Liu, Solvability of Boundary Value Problems for Singular Quasi-Laplacian Differential Equations on the Whole, Mathematical Modelling and Analysis, 17(3)(2012) 423-446.

[18] Y. Liu, Existence of solutions of boundary value problems for coupled singular differential equations on whole lines with impulses, Mediterr. J. Math. DOI 10.1007/s00009-014-0422-1, to appear.

[19] J.D. Murray, Mathematical Biology, Springer-Verlag, Berlin, 1993.

[20] R.A. Fisher, The wave of advance of advantageous genes, Ann. Eugenics 7(1937) 353-369.

[21] C. Marcelli, Existence of solutions to some boundary-value problems governed by general nonlinear non-autonomous differential operators, Electron. J. Differ. Equ. 2012, 171 (2012).

[22] C. Marcelli, The role of boundary data on the solvability of some equations involving non-autonomous nonlinear differential operators, Boundary Value Problems, 2013, 2013: 252 .

[23] P.M. Mcabe, J.A. Leach and D.J. Needham, The evolution of travelling waves in fractional order autocatalysis with decay. I. Permanent form travelling waves, SIAM J. Appl. Math., 59 (1998) 870-899.

[24] C. Marcelli, F. Papalini, Heteroclinic connections for fully non-linear nonautonomous second-order differential equations, Journal of Differential Equations, 241(2007) 160-183.

[25] K. R. Prasad, A. K. Rao, S. N. Rao, Existence of positive solutions for the system of higher order two-point boundary value problems, Proceedings of the Indian Academy of Sciences Mathematical Sciences, 122(2012) 139-152.

[26] C. G. Philos, I. K. Purnaras, A boundary value problem on the whole line to second order nonlinear differential equations, Georgian Mathematical Journal, 17(2010) 241252 .

[27] A. Qian, C. Li, Infinitely Many Solutions for a Robin Boundary Value Problem, International Journal of Differential Equations Volume 2010 (2010), Article ID 548702, 9 pages. 
[28] J. Sun, Nonlinear Functional Analysis and Its Application. Science Press, Beijing (in Chinese) (2008).

[29] S. Shi and S. Li, Existence of solutions for a class of semilinear elliptic equations with the Robin boundary value condition, Nonlinear Analysis: Theory, Methods and Applications, vol. 71, no. 7-8, pp. 3292-3298, 2009.

[30] H. Sun, Y. Li, J. J. Nieto, Q. Tang, Existence of Solutions for Sturm-Liouville Boundary Value Problem of Impulsive Differential Equations, Abstract and Applied Analysis Volume 2012 (2012), Article ID 707163, 19 pages.

[31] Y. Tian and W. Ge, Variational methods to Sturm-Liouville boundary value problem for impulsive differential equations, Nonlinear Analysis, vol. 72, no. 1, pp. 277-287, 2010 .

[32] Y. Tian and W. Ge, Multiple solutions of impulsive Sturm-Liouville boundary value problem via lower and upper solutions and variational methods, Journal of Mathematical Analysis and Applications, vol. 387, no. 2, pp. 475-489, 2012.

[33] X. Yang, Y. Liu, Existence of unbounded solutions of boundary value problems for singular differential systems on whole line, Boundary Value Problems, 2015(2015) 42: 1-39.

[34] H. Zou, A priori estimates for semilinear elliptic system without variational structure and their applications, Math. Ann. 323(2002) 713-735.

\author{
XiaOHU Yang \\ Department of Computer \\ Guangdong Police College \\ 510230 GuAngzhou \\ People's Republic of China
}

YuJi LiU

Department of Mathematics

Guangdong University of Finance and Economics 510320 Guangzhou

People's Republic of China

E-mail address: liuyuji888@sohu.com 